\title{
Bericht vom Trustee Meeting 2016 der AO-Stiftung in Amsterdam
}

$\square$ Christian Voigt

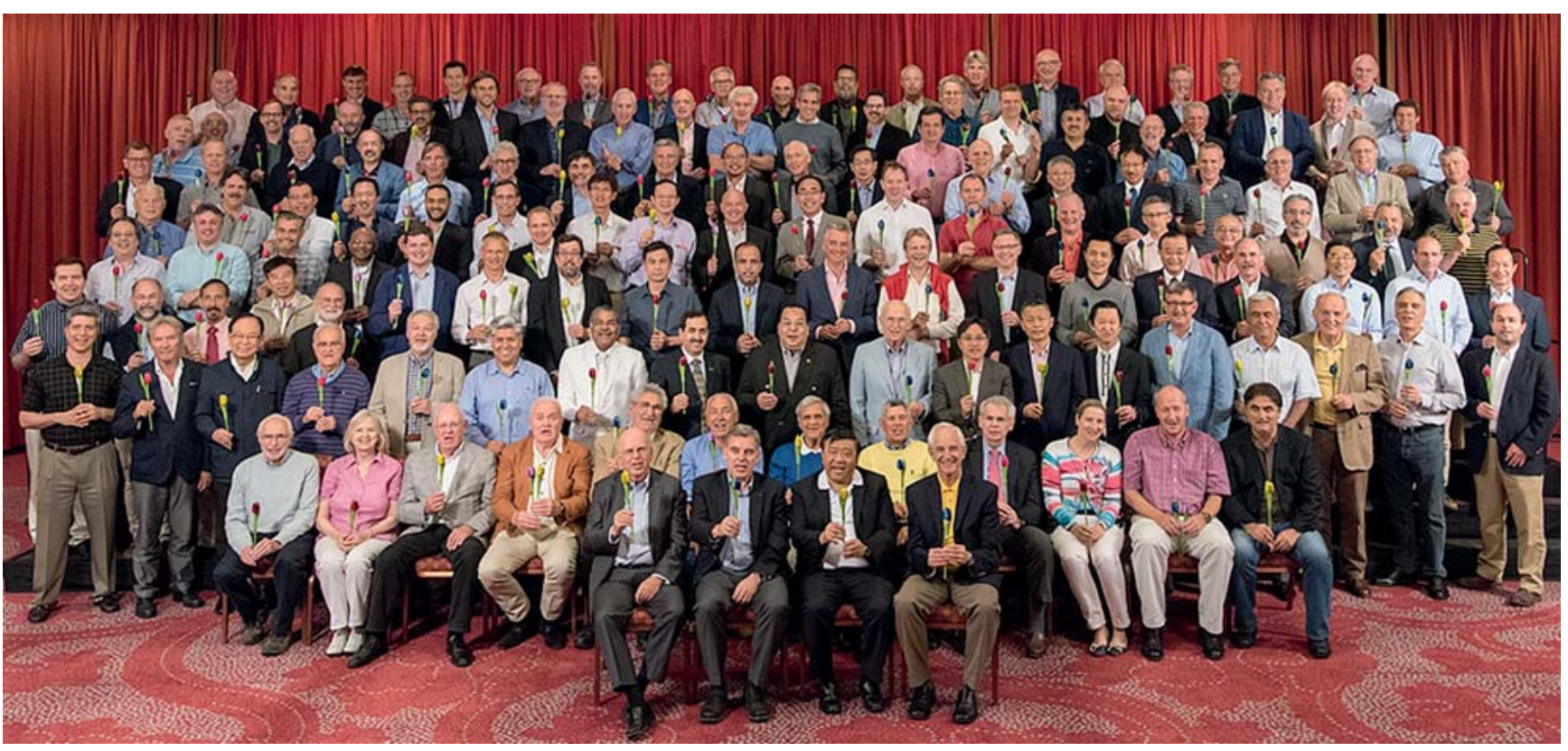

Abb. 1 Gruppenfoto der AO Trustees. Foto: Jürgen Staiger, mit freundlicher Genehmigung der AO Foundation.

Die Versammlung der Trustees ist das AO-Parlament. Es ist verantwortlich für die wissenschaftlichen und klinischen Aufgaben der AO Foundation. Es wählt die Mitglieder der obersten Gremien der AO und kann über die Satzung entscheiden. Die Trustees werden von ihren jeweiligen Regionen (Europa, Nordamerika, Lateinamerika, Asien/Pazifik und Mittlerer Osten) und dabei nochmals in den jeweiligen Gruppen (AOTrauma, AOSpine, AOCMF, AOVet, AONeuro) gewichtet vorgeschlagen und dann von der Versammlung der Trustees für 5 Jahre gewählt. So ist gewährleistet, dass eine ständige Erneuerung des „Parlaments“ erfolgt. Anlässlich der diesjährigen Versammlung wurden 19 Trustees neu gewählt, 30 wurden verabschiedet. Deutschland stellt insgesamt 16 Trustees: Carl-Peter Cornelius (München, MKG),

OP-JOURNAL 2016; 32: 165-167

(c) Georg Thieme Verlag KG Stuttgart · New York DOI http://dx.doi.org/10.1055/s-0042-115790
Florian Gebhard (Ulm), Norbert Haas (Berlin, ehem. Präsident), Christoph Josten (Leipzig), Frank Kandziora (Frankfurt, Wirbelsäule), Philipp Lobenhoffer (Hannover), Gerson Mast (München, MKG), Michael Nerlich (Regensburg), Tim Pholemann (Homburg), Michael Raschke (Münster), Klaus Schnake (Fürth, Wirbelsäule), Michael Schütz (Berlin), Ulrich Stöckle (Tübingen), Christian Voigt (Solingen), Sigfried Weller (Tübingen, ehem. Präsident), Karsten Wiechert (Augsburg, Wirbelsäule).

Das diesjährige Treffen - das 36. seit dem 1. Treffen 1984 - fand vom 7.-10. Juli 2016 im Okura Hotel in Amsterdam statt. Das Programm war straff getaktet: Sitzungsbeginn 7:00 bzw. 8:00 Uhr, Ende 17:00 Uhr, dazwischen ein kurzer Lunch.

Am 1. Tag wurden wir auf Amsterdam und die Niederlande eingestimmt. Nach einem kurzen Beitrag über den Werdegang der niederländischen AO folgte ein interessanter Gastvortrag des europä- ischen Astronauten, der am längsten (193 Tage) im All gewesen ist: André Kuipers, ein Arzt. Er informierte uns über Auswahlkriterien und Training zum Astronauten, über die Arbeit im Space Lab und die Wirkung des langen Raumflugs mit verminderter Gravitation auf den menschlichen Körper.

Dann verfolgte das etwas merkwürdig anzusehende Auditorium mit 3-D-Brillen den interessanten Beitrag des jungen Chapters AONeuro über innovative Techniken bei der Operation an der Schädelbasis.

Die Sitzung über die Innovationen der AO schloss dann das Programm des 1 . Vormittags.

Am Nachmittag nahmen die Trustees dann ihre eigentlichen Aufgaben wahr mit Abstimmungen und Entlastungen des AO Foundation Board (AOFB) und der Bestätigung der Wahl des nächsten Präsidenten der AO Foundation Nikolaus 


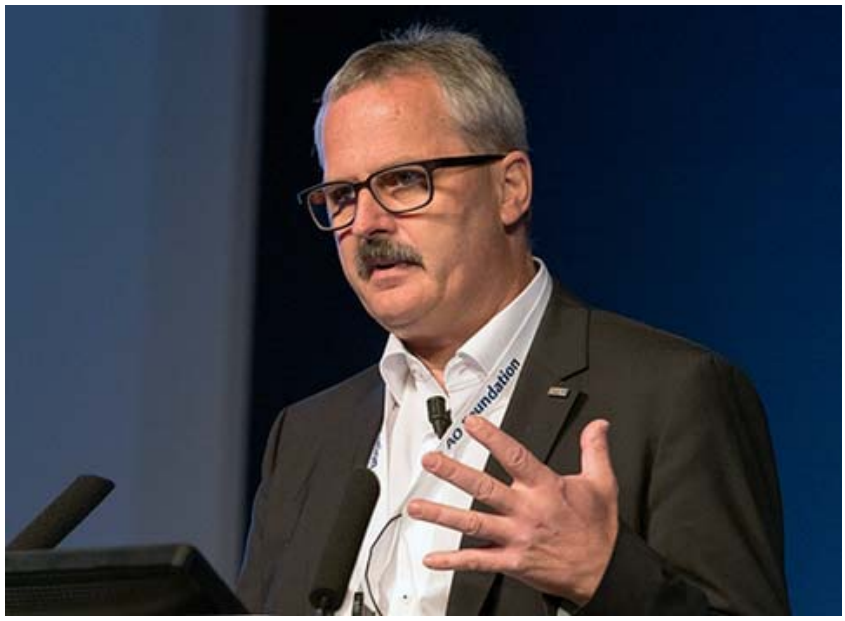

Abb. 2 Trustee Florian Gebhard, Ulm, während eines Vortrags. Foto: Jürgen Staiger, mit freundlicher Genehmigung der AO Foundation.

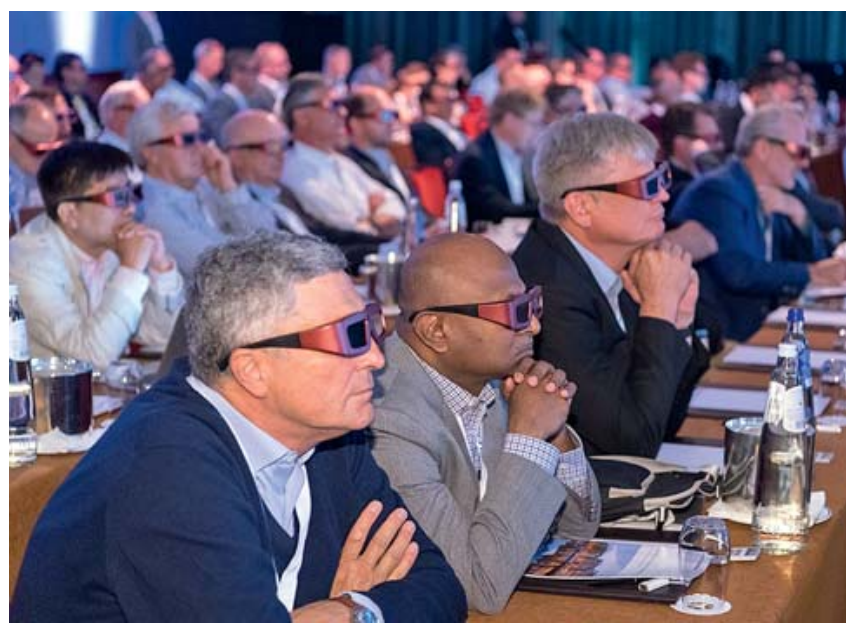

Abb. 4 Auditorium verfolgt AONeuro-Vortrag. Foto: Jürgen Staiger, mit freundlicher Genehmigung der AO Foundation.

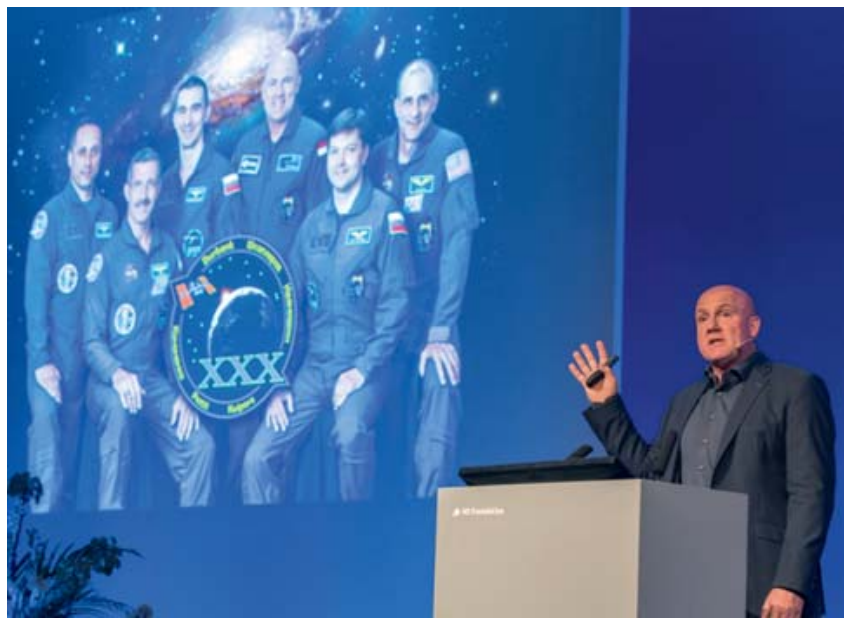

Abb. 3 Astronaut André Kuipers. Foto: Jürgen Staiger, mit freundlicher Genehmigung der AO Foundation.

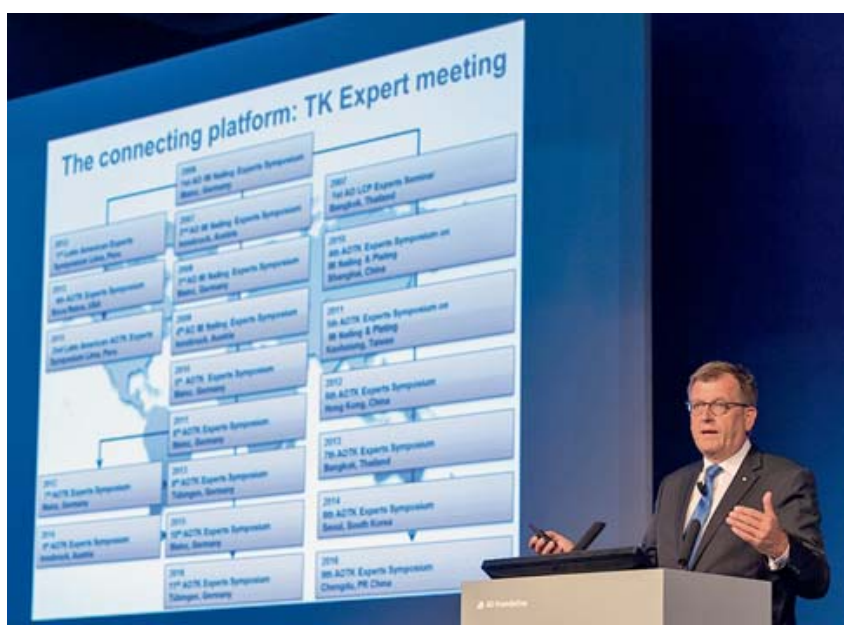

Abb. 5 Trustee Tim Pohlemann, Homburg/Saar, referiert über Innovationen. Foto: Jürgen Staiger, mit freundlicher Genehmigung der AO Foundation.
Renner, Schweiz, und der Wahl des President-elect Robert McGuire, USA.

Am 2. Tag fanden zunächst Parallelsitzungen der jeweiligen Chapters der AO statt. Bei AOTrauma ging es in einer der beiden Gruppen um die „relevante und proaktive Kommunikation“ innerhalb des Chapters - via Brief, E-Mail, Social Media etc. einschließlich der Frage der Frequenz.

Am Nachmittag befasste sich eine große Sitzung mit 7 Sprechern mit dem möglichen Beitrag der AO im Rahmen des sich ändernden gesundheitsökonomischen Umfelds. Daran schlossen sich Beiträge über die Rolle der Trustees innerhalb des Ethikkodexes der AO und über akademische Plagiate an.

Am letzten Tag begannen die Sitzungen mit einem Beitrag über die weit gefassten sozialen Verantwortlichkeiten der

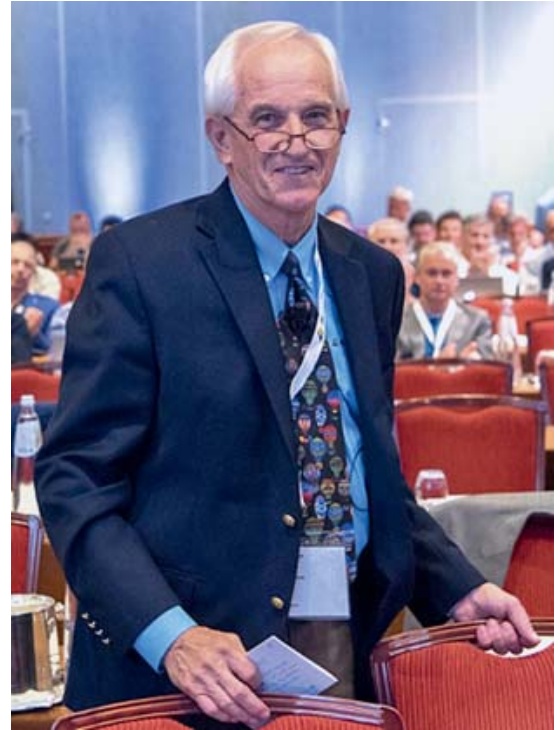

Abb. 6 Robert McGuire direkt nach seiner Wahl zum President-elect. Foto: Jürgen Staiger, mit freundlicher Genehmigung der AO Foundation.

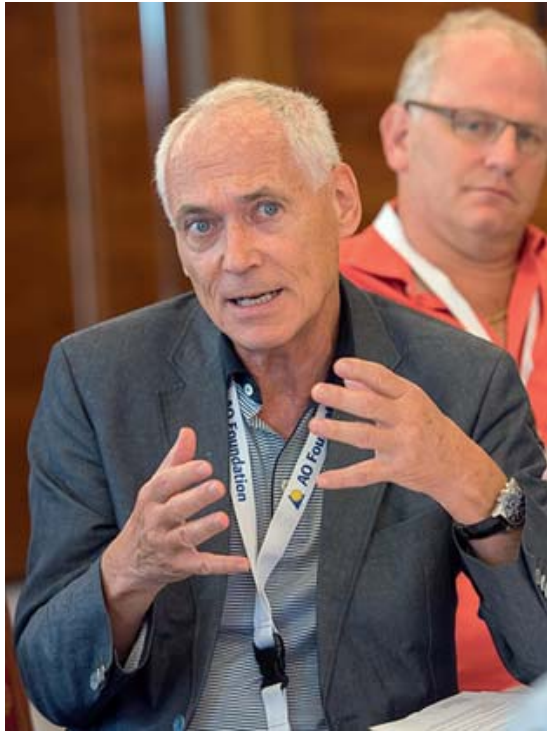

Abb. 7 Trustee Christian Voigt, Solingen, während einer Diskussion. Foto: Jürgen Staiger, mit freundlicher Genehmigung der AO Foundation. 


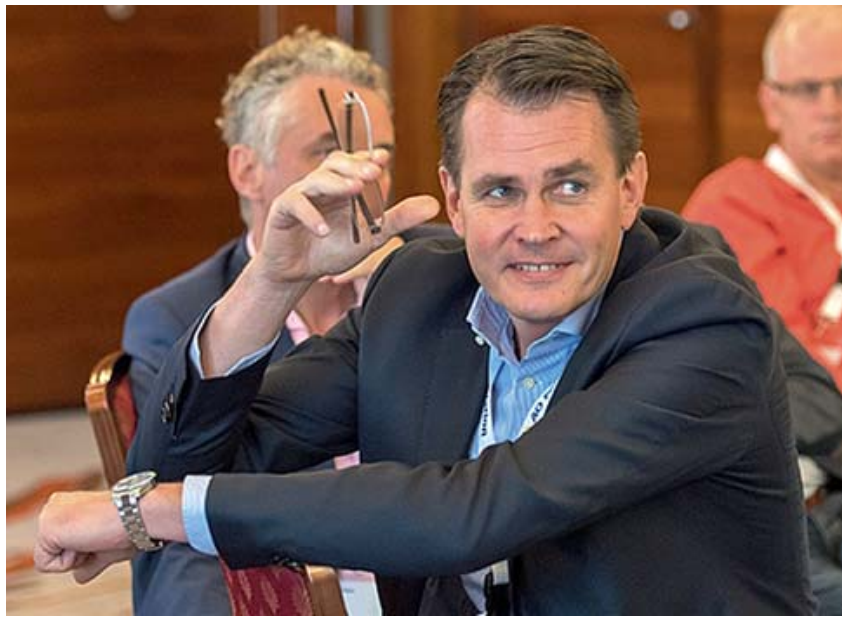

Abb. 8 Trustee Ulrich Stöckle, Tübingen. Foto: Jürgen Staiger, mit freundlicher Genehmigung der AO Foundation.

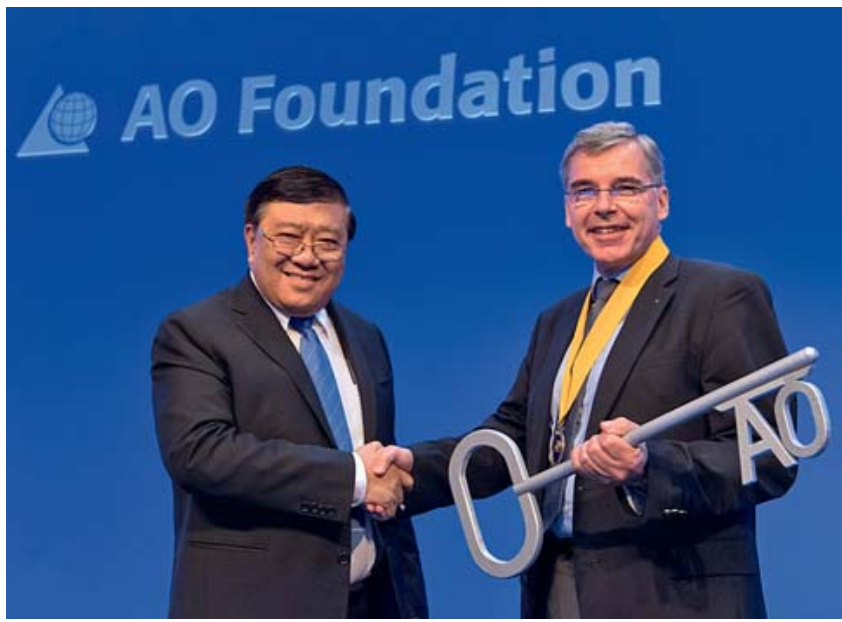

Abb. 10 Suthorn Bavonratanavech, Thailand, übergibt den symbolischen Schlüssel der AO an Klaus Renner, Schweiz. Foto: Jürgen Staiger, mit freundlicher Genehmigung der AO Foundation.

AO selbst - von einem Anteil von 53,7\% weiblichen Angestellten über eine Verminderung des Gesamtenergieverbrauchs seit 2007 von 50\% u. Ä. Es folgten dann die Rechenschaftsberichte je- des AO-Instituts und des AO Strategy Fund. Danach stellte der Gewinner des Berton-Rahn-Forschungspreises seine Arbeit vor. Von AOVet gab es einen Beitrag über die Endoprothetik der Hüfte beim Hund und deren Rückschlüsse auf den Menschen. Einen sehr guten Vortrag gab es über das Thema „Die Kunst der Wissensvermittlung bei der Translation von Forschungsergebnissen in den klinischen Alltag“.

Das Treffen schloss ab mit einer sehr emotionalen Rede des scheidenden Präsidenten Suthorn Bavonratanavech, Thailand, über sein Leben in und mit der AO und über seine Präsidentschaft, bevor er den Staffelstab an den nächsten Präsidenten Nikolaus Renner, Schweiz, weitergab. Dieser schilderte kurz seine Pläne während seiner Präsidentschaft und lud ein zum nächsten Treffen der Trustees im Juli 2017 in Miami, USA.

\section{Dr. Christian Voigt}

AO Trustee

Solingen 